\title{
Advanced Microscopic Characterisation through Integrated Learning Tools
}

\author{
B. Cribb, T. White, J. Shapter, J. Muhling, L. Soon, S.P. Ringer, E. Grinan, C. Frost and P.R. Munroe \\ Australian Microscopy and Microanalysis Research Facility, Sydney, NSW2006, Australia.
}

The Australian Microscopy and Microanalysis Research Facility (AMMRF) is a national nodal network that acts as the peak body for microscopy, and related modalities, in Australia. One of the principal roles of AMMRF is to prosecute programs in research training in microscopy. Currently, much of this training is provided either in the classroom or through one-to-one training at the instrument itself.

However, there are deficiencies in this approach as traditional pedagogies do not optimally serve the needs of a rapidly growing student body needing such training, moreover, the diversity of this cohort, ranging from undergraduates through to researchers and technical staff, cannot be adequately addressed within current educational models.

The AMMRF recently secured funding from the Australian Learning and Teaching Council (ALTC) to develop a flexible and comprehensive blended learning program to create an enhanced education landscape available to those wishing to learn microscopy. We have undertaken to create a national bank of learning tools (e-teaching modules) that provide a new kind of education landscape that integrates with traditional learning environments (see Fig. 1). For students this approach will increase the depth of knowledge they attain and accelerate their rate of learning. Importantly, it will also allow students to find individualised pathways to learning end-points and to undertake specialization of skills. The modules developed build on the Online Micro and Nanocharacterisation Instruction (OMNI) approach developed by one of us, White, whilst at the Nanyang Technological University, Singapore [1].

As shown in figure 1, present practice is to move directly from Stage 1 (Introduction) to Stage 3 (Training). However, many students are ill-prepared for hands-on experience, causing frustration for them and teachers alike. This project is developing Stage 2 through blended learning modules, on a technique-by-technique basis, to provide a greater depth and breadth of theoretical and practical knowhow. A guided and comprehensive route towards advanced competency will reach out to new students and provide a time-effective path for researchers and managers wishing to upgrade their skills. This approach also greatly expands the cohort size that can be accommodated.

We are constructing e-learning modules for characterisation techniques that will reside at the core of a novel blended learning environment. Modules are being developed for scanning electron microscopy (SEM), transmission electron microscopy (TEM), microanalysis, $x$-ray diffraction (XRD), atomic force microscopy (AFM) and light microscopy. Each module is self-contained and composed of a package of interactive e-tools that introduce the student to physical principles, specimen selection and preparation, operation guides, and machine simulations. The modules are flexible, allowing students to explore at their own pace topics that interest them. The modules allow tailored pathways that best meet student aspirations, and allow researchers to select the correct instruments to deliver the data required. Students may take automated on-line tests that provide timely on-line formative assessments and comprehensive summative assessment that provide feedback on module tools to be re-visited and act as gateways before proceeding to more challenging topics. 
Central to each of the modules is a virtual instrument. Figure 2 shows a screen grab of the virtual SEM. A number of other simulated instruments, either SEM's or AFM's, have been recently developed, however, these are often limited in functionality, appearing more for demonstration purposes rather than as an integrated educational system [2-5]. Our virtual machine simultaneously shows what occurs in the mechanics of the machine alongside the effect on the image. It provides a wide range of magnifications and uses image-based assessment. Small download sizes keep usability to a maximum.

\section{References}

[1] http://webserver.mse.ntu.edu.sg/omni/

[2] http://www.virtualsem.com/

[3] http://education.denniskunkel.com/JavaScriptSEM.php

[4] http://micro.magnet.fsu.edu/primer/java/electronmicroscopy/magnify $1 /$ index.html

[5] http://www.virlab.virginia.edu/VL/SEM.htm; http://virlab.virginia.edu/VL/easyScan_AFM.htm

[6] Support for this work has been provided by the Australian Learning and Teaching Council Ltd, an initiative of the Australian Government Department of Education, Employment and Workplace Relations. The views expressed in this publication do not necessarily reflect the views of the Australian Learning and Teaching Council.

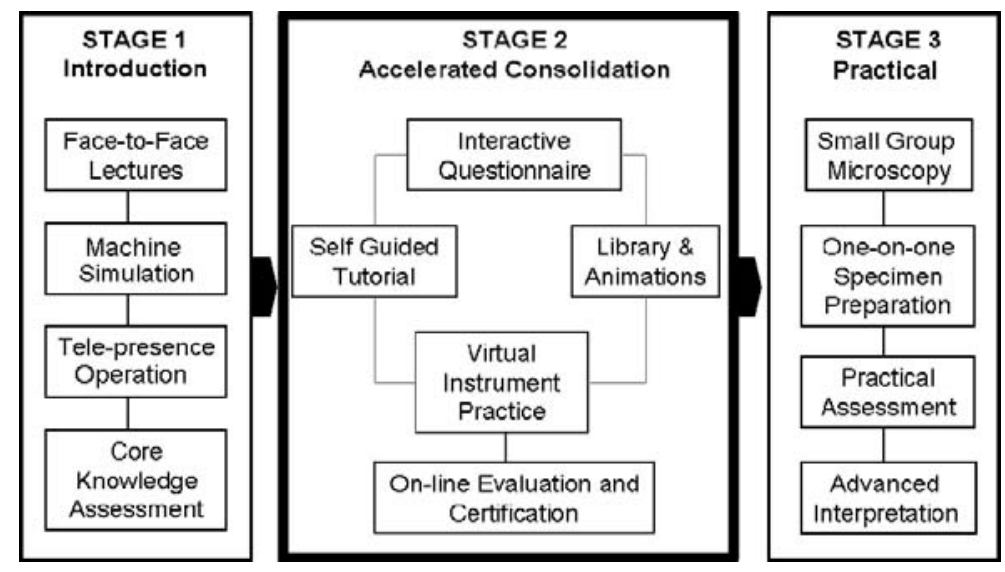

Fig. 1. The enhanced blended learning environment for education in characterisation techniques

Fig 2. The SEM simulator

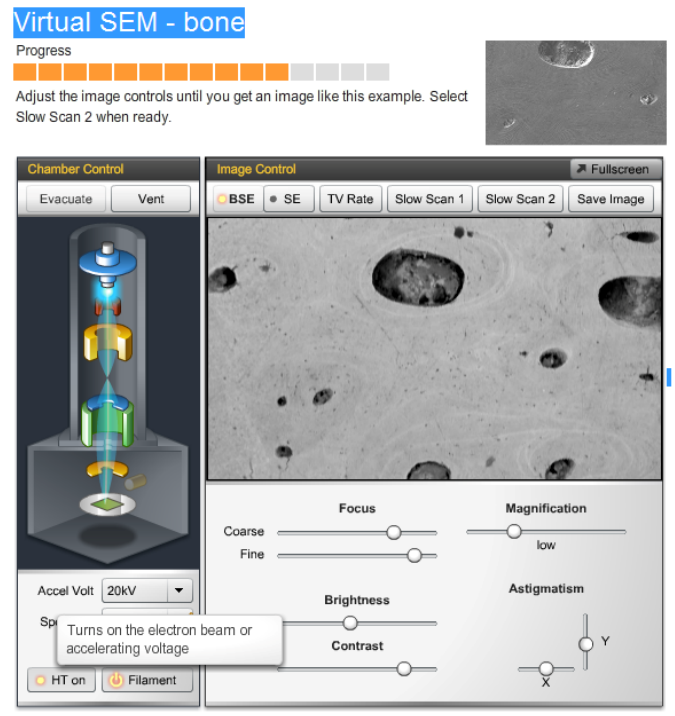

